\title{
Implicit Spatial Length Modulates Time Estimates, But Not Vice Versa
}

\author{
Roberto Bottini ${ }^{1,2}$ and Daniel Casasanto ${ }^{2,3}$ \\ ${ }^{1}$ University of Bergamo, Department of Human Sciences, Bergamo, IT \\ ${ }^{2}$ Max Planck Institute for Psycholinguistics, Neurobiology of Language Group, \\ Nijmegen, NL \\ ${ }^{3}$ Donders Center for Brain, Cognition, and Behavior, Radboud University, Nijmegen, NL \\ roberto.bottini@unibg.it \\ daniel.casasanto@mpi.nl
}

\begin{abstract}
How are space and time represented in the human mind? Here we evaluate two theoretical proposals, one suggesting a symmetric relationship between space and time (ATOM theory) and the other an asymmetric relationship (metaphor theory). In Experiment 1, Dutch-speakers saw 7-letter nouns that named concrete objects of various spatial lengths (tr. pencil, bench, footpath) and estimated how much time they remained on the screen. In Experiment 2, participants saw nouns naming temporal events of various durations ( $t r$. blink, party, season) and estimated the words' spatial length. Nouns that named short objects were judged to remain on the screen for a shorter time, and nouns that named longer objects to remain for a longer time. By contrast, variations in the duration of the event nouns' referents had no effect on judgments of the words' spatial length. This asymmetric pattern of cross-dimensional interference supports metaphor theory and challenges ATOM.
\end{abstract}

Keywords: ATOM, Metaphor, Psychophysics, Space, Time.

\section{Introduction}

Space and time are intimately related in the human mind, as they are in the physical world. But exactly how are these dimensions related? Here we evaluate two theoretical proposals, one suggesting a symmetric and the other an asymmetric relationship between space and time.

According to the first proposal, space and time are represented in the brain and mind by a common analog magnitude system, which also generates representations of number and quantity. This view, summarized in Walsh's ATOM (A Theory of Magnitude; [1]), is consistent with neurological data showing shared brain areas for processing space, time, and quantity [e.g. 2], and with many behavioral studies in animals and humans [e.g., 3, 4, 5, 6].

Implicit in ATOM is an assumption that these 'ATOMic' dimensions are symmetrically interrelated: not hierarchically related in the brain/mind. Accordingly, Walsh [1] frames predictions in symmetrical terms, positing "overlapping brain regions" and "cross-domain, within-magnitude priming" between dimensions, without specifying 
any directionality to the priming (or interference) effects. Indeed, if space and time are both manifestations of the same general-purpose analog magnitude system, there may be no a priori reason to posit that one dimension should depend asymmetrically on another.

On an alternative proposal, space, time, and quantity are importantly related, but in a different way. According to theories of metaphorical mental representation [e.g., 7], representations of time, number, and quantity depend asymmetrically on representations of space. The claim that some domains are asymmetrically dependent on others, which is at the core of metaphor theory, was originally supported by patterns in metaphorical language. In English, it is nearly impossible to talk about domains like time without using words whose primary meaning is spatial (denotatively, developmentally, or historically [8]). Vacations can be long or short, meetings can be moved forward or pushed back, deadlines can loom ahead or lie behind us. Yet, it is far less common to use temporal words to talk about space [7]. This asymmetry in language has been echoed by behavioral findings in psycholinguistics [9], cognitive development, [10], and psychophysics [11].

In one set of studies by Casasanto \& Boroditsky, participants viewed lines of various spatial lengths that appeared on a screen for varying durations [11]. They were asked to estimate either the duration or the spatial length of each line, using mouse clicks. Participants were unable to ignore irrelevant spatial information when making judgments about duration, but not the converse. For stimuli of the same average duration, lines that extended shorter in space were judged to take a shorter time, and lines that extended longer in space were judged to take a longer time. By contrast, for stimuli of the same average spatial length, spatial estimation was not affected by the line's duration. This cross-dimensional asymmetry, predicted based on patterns in language, was shown here in non-linguistic psychophysical judgments. Five follow-up experiments varied the attentional, mnemonic, and perceptual demands of the stimuli, and all six experiments supported the same conclusion: mental representations of time depend on representations of space, more than vice versa.

This robust space-time asymmetry supports metaphor theory, but presents a challenge to ATOM. If space and time are both derived from (or are both manifestations of) a general-purpose magnitude metric, then why should representations of time depend on representations of space more than the other way around -- in adults and children, and in language and thought?

It might be possible to reconcile these results with ATOM by positing that in previous studies, space influenced time asymmetrically because space was either (a) the more discriminable dimension, or (b) the more perceptually salient dimension in the stimulus. Discriminability, in this context, refers to the resolution at which a dimension is sampled. Salience means the extent to which one dimension attracts attention relative to the other. Differences in discriminability and perceptual salience have been shown to modulate the strength or direction of cross-dimensional interference and priming effects across numerous studies [12]. In general, the dimension that is more discriminable or salient interferes with the dimension that is less discriminable or less salient. Can task-related differences in the relative discriminability or salience of stimulus dimensions account for the space-time asymmetries observed previously?

One set of studies reviewed above addressed these questions. Tests of crossdimensional relationships often manipulate more levels of one dimension than of the 
other, creating an imbalance in discriminability [13]. In the space-time experiments by Casasanto \& Boroditsky [11], however, there were 9 levels of each dimension fully crossed, to equate discriminability.

Differences in discriminability may correspond to differences in the accuracy, precision, or variability of judgments across domains. This complicates the interpretation of cross-dimensional interference effects. In the limit, if performance in one domain is perfect, there is no opportunity for variation in the other domain to influence it: the 'clean' domain can influence performance in the 'messy' domain, but not vice versa. In Casasanto \& Boroditsky's studies, however, within-domain performance was equivalent across space and time [see also 10].

But is it possible that space was more salient than time in these studies? Following Garner [14], Casasanto \& Boroditsky [11] asked participants to judge different dimensions of the same stimuli (e.g., the spatial or temporal extent of a line). Thus, people had the exact same perceptual input during space and time judgments. But this does not guarantee that the dimensions were equally perceptually salient: it is possible to see the spatial extent of a line, but not its duration. To address the concern that space may have been more salient than time, in one experiment each line was accompanied by a tone, which sounded for the duration that the line remained on the screen. Tones have temporal extent but no spatial extent. Thus, temporal information was available to the participant through two sensory channels, but spatial information through only one. Yet, increasing the salience of temporal information did not diminish the space-time asymmetry.

Still, on a skeptical interpretation, these previous studies may not have ruled out cross-dimensional differences in perceptual salience definitively. It is possible that space will always be more perceptually salient than time whenever perceptible spatial stimuli are used, since it is possible to perceive space, but arguably it is not possible to perceive time directly through the senses [15]. The question remains, then, whether the space-time asymmetry would persist in psychophysical judgments if differences in the perceptual salience of space and time in the stimulus were eliminated.

In the present study, we eliminated differences in perceptual salience by eliminating perceptible variation in the critical dimension (space or time), altogether. We tested whether the implicit spatial information encoded in object nouns can influence estimates of time (in Experiment 1), and whether the temporal information encoded in event nouns can influence estimates of spatial length (in Experiment 2). Participants saw words presented one at a time and reproduced either the duration for which they remained on the screen or their spatial length, using mouse clicks as in Casasanto \& Boroditsky [11]. In the duration estimation task (Experiment 1), the target words named objects of various spatial lengths (e.g., pencil, clothesline, footpath). All target words had the same number of letters in Dutch, and therefore the same physical length on the screen. In the spatial length estimation task (Experiment 2) the target words named events of various durations (e.g., blink, party, season). Again, all target words had the same number of letters, but they were presented with a varying number of spaces between letters (1-9 spaces), stretching them out to different spatial lengths on the screen.

Word meanings were irrelevant to the length and duration estimations. We expected, however, that participants would read the words while viewing them, and activate their meanings (voluntarily or involuntarily). Presumably, the meaning of an 
object noun typically includes a representation of the object's spatial form, and the meaning of an event noun a representation of the event's duration. If internally generated spatial and temporal representations cued by words are sufficient to modulate estimates of experienced duration and spatial length, then we should observe cross-dimensional interference. Following metaphor theory, we predicted that the cross-dimensional interference should be asymmetric, even in the absence of crossdimensional differences in perceptual salience: spatial representations cued by object nouns should modulate estimates of their duration more than temporal representations cued by event nouns modulate estimates of their spatial extent on the screen.

\section{Does Implicit Spatial Length Modulate Time Estimates?}

Experiment 1 tested whether the spatial length of a word's referent can modulate estimates of how much time the word remained on the screen.

\subsection{Methods}

Participants. Native Dutch-speakers $(\mathrm{N}=39)$ performed Experiment 1 in exchange for payment.

Materials. Dutch nouns naming 9 concrete objects (Targets) and 9 abstract entities (Fillers) were presented on a computer monitor (resolution $=1024 \times 768$ pixels) for varying durations. The concrete nouns referred to objects whose characteristic spatial lengths ranged from short (normally measured in centimetres) to long (normally measured in kilometres). English equivalents of these nouns are listed here in order of increasing length: cigarette, pencil, ruler, meter stick, bench, clothesline, footpath, lane, highway. In Dutch, all 9 target nouns had 7 letters, and were presented on the screen in a fixed-width font (62-point Courier New). Therefore, the targets did not differ in their physical spatial lengths on the screen; rather, they differed in their implicit lengths (i.e., the typical spatial lengths of their referents).

The filler nouns referred to abstract entities that have no physical spatial length: guess, idea, pride, opinion, envy, thought, philosophy, suspicion, dignity. However, they varied in their number of letters in Dutch (from 3-11 letters) and therefore in their physical length on the screen (nine different lengths, varying from 50-450 pixels as measured from the left edge of the first letter to the right edge of the last letter). By contrast with the targets, the fillers did not differ in the implicit lengths of their referents; rather, they differed in their physical lengths on the screen.

Each target and filler word was presented 9 times throughout the experiment, for 9 different durations. Durations ranged from 1000 to $5000 \mathrm{~ms}$ in $500 \mathrm{~ms}$ increments. Fully crossing these 9 durations with the target words (which had 9 different implicit spatial lengths) produced 81 target trials. Likewise, fully crossing the 9 durations with the filler words (which had 9 different physical lengths on the screen) produced 81 filler trials. The 162 different trials were presented in random order, with fillers and targets intermixed. Words were presented in white letters on a black background in the center of the screen. Participants were tested individually and testing lasted about 30 minutes. 
Procedure. Participants viewed the 162 words, one word at a time, from a viewing distance of approximately $50 \mathrm{~cm}$. Immediately after each word disappeared an "hourglass" icon appeared in the upper left corner of the monitor indicating that the subject should reproduce the amount of time the word remained on the screen. To estimate duration, subjects clicked the mouse once on the center of the hourglass, waited the appropriate amount of time, and clicked again in the same spot, thus indicating the beginning and end of the temporal interval. All responses were self-paced. After the experiment there was a two-part debriefing. In the first part, the experimenter asked the participant "What do you think this experiment is about?" and "What do you think we were looking for?" to determine whether the participant was aware of any relationship between the implicit lengths of the target words and their durations. In the second part, participants saw each target word again, in random order, and verbally estimated the typical spatial length of the target words' referents (using an appropriate unit of measurement). These subjective length estimates were used in later analyses as predictors of subjective duration.

\subsection{Results and Discussion}

Four participants were removed from the analyses below: one for giving nonsensical answers in the debriefing, one for excessively poor time estimation performance according to the criterion used by Casasanto \& Boroditsky [11] ${ }^{1}$, and two for guessing that there was a connection between the meanings of the target words and time estimation.

For the remaining 35 participants, we first analyzed participants' duration estimates as a function of the actual duration of the stimuli. Overall, duration estimates for target words were highly accurate (mean effect of actual duration on estimated duration: $\mathrm{y}=0.83 \mathrm{x}+154.11, \mathrm{r}^{2}=.99, \mathrm{df}=7, \mathrm{p}<.001$; fig 1a).

We then tested for effects of implicit length on duration estimation. Target words were rank-ordered according to the typical lengths of their referents (this a priori ranking was confirmed by participants' post-test length estimates). Non-parametric correlation showed that implicit spatial length affected estimates of duration $(\mathrm{y}=3.77 \mathrm{x}$ $+2605.70, \mathrm{r}_{\mathrm{s}(\text { Spearman's sho })}=0.75, \mathrm{df}=7, \mathrm{p}<.001$; fig. $1 \mathrm{~b}$ ).

Finally, we conducted a parametric analysis of the effect of implicit length on duration estimation. Participants' post-test ratings of the typical spatial length of each target word's referent were used as a predictor of their duration estimates. Ratings for each target item were averaged, and the average length estimates in meters were transformed by a base 10 logarithm. This analysis corroborated the non-parametric analysis, showing a highly significant effect of implicit spatial length on duration estimation $\left(\mathrm{y}=5.60 \mathrm{x}+2619.20, \mathrm{r}^{2}=.57, \mathrm{df}=7, \mathrm{p}<.001\right)$.

Participants incorporated irrelevant spatial information into their temporal estimates. For stimuli of the same average duration, words with (spatially) shorter referents were judged to remain on the screen for a shorter time, and words with longer referents for a longer time. This was true even though the task did not require participants to process the words' meanings.

${ }^{1}$ Participants were excluded if the slope of their within-domain duration or length estimates was less than 0.5 [see, 11]. This criterion, which resulted in the exclusion of only one participant overall, is unbiased with respect to the predicted cross-dimensional interference because length and duration are orthogonal in the designs of both experiments. 

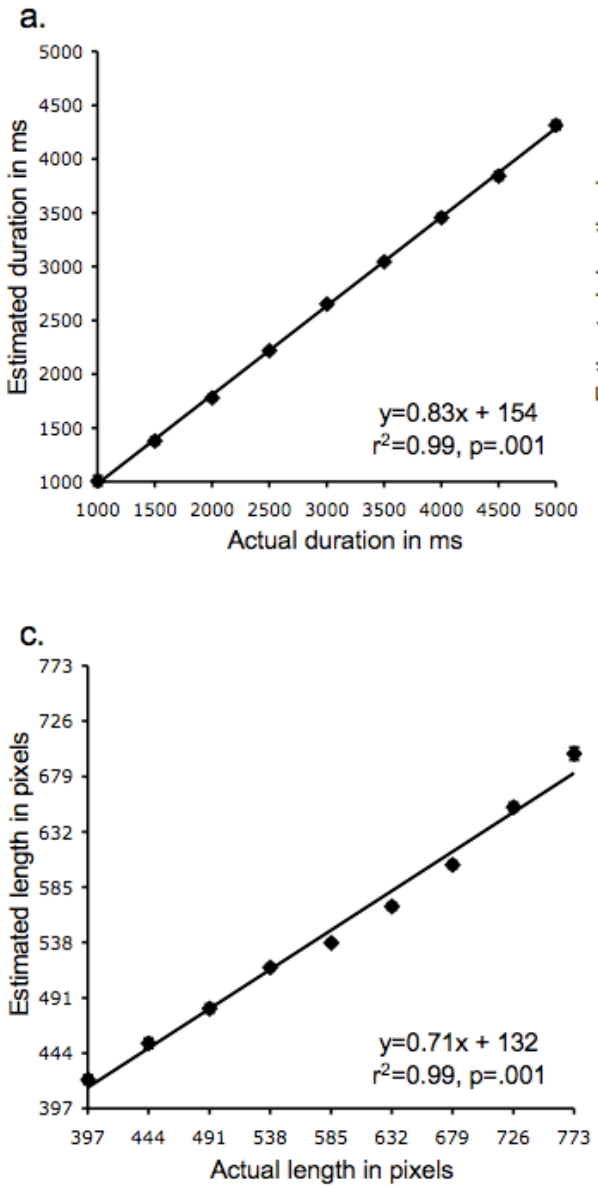

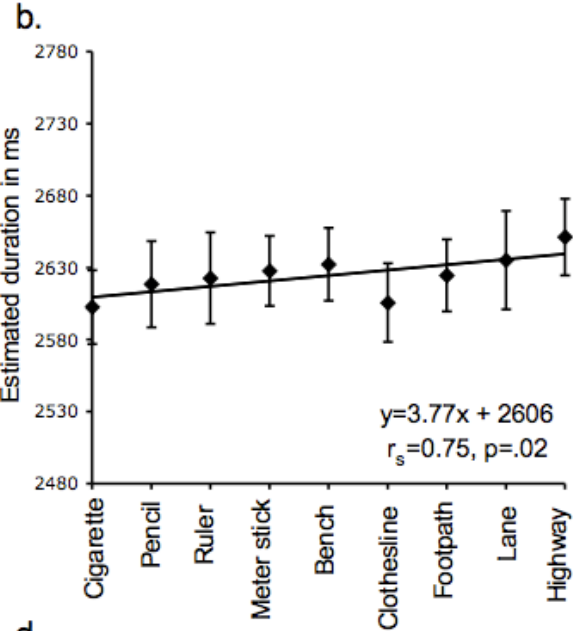

d.

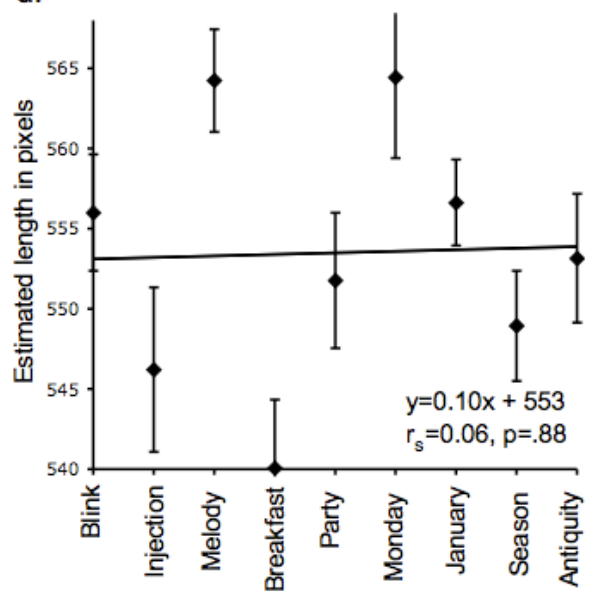

Fig. 1. Results of Experiment 1 (top) and Experiment 2 (bottom). 1a. Within-domain effect of actual word duration on estimated duration. 1b. Cross-domain effect of words' implicit spatial length on estimated duration. 1c. Within-domain effect of actual word length on estimated spatial length. 1d. Cross-domain effect of words' implicit duration on estimated spatial length. The axes of the top and bottom plots (a-c, b-d) are proportional with respect to the total range of target values. Error bars show s.e.m.

This result shows that perceptible spatial input is not necessary to modulate time estimates; rather, internally- generated spatial representations cued by words are sufficient. This outcome, per se, is equally consistent with metaphor theory and with ATOM. To distinguish between the theories, it is necessary to conduct a complementary experiment to determine whether implicit duration can affect estimates of spatial length, and whether cross-dimensional interference effects are as symmetric, as expected on ATOM (Effect of Space on Time $\approx$ Effect of Time on Space) or asymmetric, as predicted by metaphor theory (Effect of Space on Time $>$ Effect of Time on Space). 


\section{Does Implicit Duration Modulate Estimates of Spatial Length?}

Experiment 2 tested whether the duration of a word's referent can modulate estimates of the word's spatial length as presented on the screen.

\subsection{Methods}

Participants. Native Dutch-speakers $(\mathrm{N}=35)$ performed Experiment 2 in exchange for payment.

Materials. Dutch nouns naming 9 events (Targets) and nine concrete objects (Fillers) were presented on a computer monitor (resolution $=1024$ x 768 pixels). The target nouns referred to events whose characteristic durations ranged from short (normally measured in seconds) to long (normally measured in years). English equivalents of these nouns are listed here in order of increasing duration: blink, injection, melody, breakfast, party, Monday, January, Season, Antiquity. All targets were presented for $3000 \mathrm{~ms}$. Therefore, the targets did not differ in the physical durations for which they remained on the screen; rather, they differed in their implicit durations (i.e., the typical durations of their referents).

The filler nouns referred to concrete objects that have no inherent duration: doormat, ballast, portrait, detritus, crystal, device, case, sawdust, handle. Each filler noun appeared for 9 different durations from 1000-5000ms, increasing in 500ms increments. By contrast with the targets, the fillers did not differ in the implicit durations of their referents; rather, they differed in the physical durations for which they remained on the screen.

In Dutch, all target and filler nouns had seven letters, and were presented on the screen in a fixed-width font (62-point Courier New). Each word was presented 9 times throughout the experiment, with a varying number of spaces in between the letters (1-9), to stretch the words out to 9 different spatial lengths on the screen. Due to the font selected, word lengths ranged from 397 to 773 pixels, in 47 pixels increments. Presenting each word at each of these 9 spatial lengths produced 81 filler trials and 81 target trials. For the fillers, spatial length was fully crossed with the physical duration for which they were presented. For the targets, spatial length was fully crossed with the implicit duration of their referents. The 162 different trials were presented in random order, with fillers and targets intermixed. Words were presented in white letters on a black background in the center of the screen. Participants were tested individually and testing lasted about 30 minutes.

Procedure. Participants viewed the 162 words, one word at a time, from a viewing distance of approximately $50 \mathrm{~cm}$. Immediately after each word disappeared an "X" appeared in the upper left corner of the monitor indicating that the subject should reproduce the spatial length that the word had occupied on the screen. To estimate length, subjects clicked the mouse once on the center of the $\mathrm{X}$, moved the mouse to the right the appropriate distance, and clicked again, thus indicating the beginning and end of a spatial interval. All responses were self-paced. After the experiment there was a two-part debriefing, as in Experiment 1. The first part was to determine whether the participant was aware of any relationship between the implicit durations of the 
target words and their spatial lengths. In the second part, participants saw each target word again, in random order, and verbally estimated the typical duration of the target words' referents (using an appropriate unit of measurement). These subjective duration estimates were used in later analyses as predictors of subjective spatial length.

\subsection{Results and Discussion}

One participant was removed from the analyses below for guessing that there was a connection between the meanings of the target words and spatial length estimation.

For the remaining 34 participants, we first analyzed participants' spatial length estimates as a function of the actual spatial length of the stimuli. Overall, length estimates for target words were highly accurate (mean effect of actual length on estimated length: $\mathrm{y}=0.71 \mathrm{x}+132.44, \mathrm{r}^{2}=.99, \mathrm{df}=7, \mathrm{p}=.001$; fig $1 \mathrm{c}$ ).

We then tested for effects of implicit duration on spatial length estimation. Target words were rank-ordered according to the typical durations of their referents (this $a$ priori ranking was confirmed by participants' post-test duration estimates). Nonparametric correlation showed that implicit duration did not affect estimates of spatial length $\left(\mathrm{y}=0.10 \mathrm{x}+553.00, \mathrm{r}_{\mathrm{s} \text { (Spearman's rho })}=0.06, \mathrm{df}=7, n s\right.$; fig. $\left.1 \mathrm{~d}\right)$.

Next, we conducted a parametric analysis using participants' post-test ratings of the typical duration of each target word's referent were used as a predictor of their length estimates. Ratings for each target item were averaged, and the average duration estimates in minutes were transformed by a base 10 logarithm. Again, there was no effect of implicit duration on spatial length estimation $\left(y=0.04 x+553.39, r^{2}=.0003\right.$, $\mathrm{df}=7, n s)$.

Finally, we compared the strength of the cross-dimensional interference effects across Experiments 1 and 2. The difference of correlations showed the predicted cross-dimensional asymmetry $\left(r_{\text {effect of spatial length on duration }}-r_{\text {effect of duration on spatial length }}=0.74\right.$, $\mathrm{z}=1.66, \mathrm{p}=0.05$, one-tailed; see fig. $1 \mathrm{~b}, 1 \mathrm{~d}$ ). This difference cannot be attributed to differences in within-domain performance $\left(\mathrm{r}_{\text {effect of actual duration on estimated duration }}-\mathrm{r}_{\text {effect of actual }}\right.$ spatial length on estimated spatial length $=0.00, \mathrm{z}=0.00, n s ;$ see fig. $1 \mathrm{a}, 1 \mathrm{c})$.

\section{General Discussion}

This study tested whether implicit spatial information encoded in concrete object nouns can influence estimates of time (in Experiment 1), and whether implicit temporal information encoded in event nouns can influence estimates of spatial length (in Experiment 2). When participants reproduced the duration for which an object noun remained on the screen, their estimates were influenced by the implicit length of the word's referent. Words that named shorter objects (e.g., cigarette, pencil) were judged to last a shorter time, and words that named longer objects (e.g., bench, highway) to last a longer time. By contrast, when participants reproduced the spatial length of an event noun, the duration of the word's referent did not influence judgments of spatial length.

This asymmetric pattern of cross-dimensional interference was predicted based on patterns in language: people talk about time in terms of space more than they talk about space in terms of time [7]. These data show that people incorporate spatial information 
into their temporal judgments even when they're not using any metaphorical language, and support the hypothesis that mental representations of time are asymmetrically dependent on representations of space: people use spatial length to think about duration, more than vice versa.

This space-time asymmetry cannot be attributed to differences in how well participants reproduced the actual durations and lengths of the stimuli, per se, since there was no significant difference between the effect of actual duration on estimated duration (fig. 1a) and the effect of actual length on estimated length (fig. 1c). Thus, differences in cross-dimensional interference were not due to differences in within-domain performance.

Furthermore, the space-time asymmetry cannot be attributed to differences in the perceptual salience of the interfering dimensions (i.e., space in Expt. 1, time in Expt. 2). In previous experiments, space could have influenced time asymmetrically because space is inherently more perceptually salient than time (which some scholars have argued can never be perceived directly [15]). But here there was no perceptible variation in the spatial component of duration-reproduction stimuli, and no perceptible variation in the temporal component of length-reproduction stimuli. Internally generated representations of spatial length, cued by words, were sufficient to modulate estimates of the words' physical duration. This was true even though the words' meanings were task-irrelevant.

Before discussing theoretical implications of these data further, it is important to consider whether the observed pattern could be due to unintended features of the stimulus words. For example, is it possible that duration estimates in Experiment 1 were influenced by implicit speed encoded in the concrete nouns, rather than implicit length? The three longest objects (footpath, lane, and highway) are all spatial paths. The speed of motion associated with these paths increases with their lengths (i.e., footpath-walking, lane-slow driving, highway-fast driving). The conflation of length and speed in these items was a consequence of restrictions on the stimuli: items had to increase in ordinal length unambiguously, and had to have 7 letters in Dutch.

If the effect of object length on duration estimates had been driven by these three items, this would be problematic. However, even a causal inspection of fig. 1b shows this was not the case. For the majority of the items there were no clear speed associations, and yet the effect of implicit length was found. For the first 5 items (cigarette, pencil, ruler, meter stick, bench), ordinal increases in implicit length corresponded to a monotonic increase in estimated duration. The predicted effect of length on duration was significant in these 5 items, alone $\left(\mathrm{y}=6.84 \mathrm{x}+2600, \mathrm{r}_{\mathrm{s} \text { (Spearman's rho })}=1.00, \mathrm{p}=.001\right)$. Thus, implicit speed was not responsible for the effect of implicit spatial length we report here (see [11], Expt. 6 for further evidence that spatial length affects duration estimates independent of speed).

On another skeptical possibility, could implicit duration encoded in object nouns have produced the observed effect on duration estimation? Looking at the longest and shortest items alone, this seems plausible. Cigarette could be associated with the time it takes to smoke a cigarette (a short time), and highway with the amount of time one typically drives on a highway (a longer time). Yet, looking at the full range of stimuli, this alternative explanation seems implausible. What durations are prepotently associated with clothesline, pencil, ruler, bench, or meter stick? Ordinal increases in spatial length predicted ordinal increases in duration estimates for 7 out of the 8 ordinal pairs 
of stimuli (i.e., cigarette < pencil; pencil < ruler; ruler < meter stick; etc.) Pairwise differences in the typical spatial lengths of the words' referents are self-evident (and were confirmed by participants' post-test ratings), but for most of these word pairs, it seems unlikely that there are corresponding pairwise differences in durations associated with the words' referents.

Finally, although the space-time asymmetry cannot be due to differences in the perceptual salience of the interfering dimensions, could they be due to differences in conceptual salience? Could the spatial component of the object words' meanings be more salient than the temporal component of the event words' meanings? We cannot rule out this possibility definitively, but this seems unlikely to be the case. It is difficult to evaluate how salient spatial length is in the meaning of bench or cigarette, and to compare this with the salience of temporal duration in the meaning of melody or party. But a few of the stimuli are very strongly associated with a unit of space (ruler, meter stick) or a period of time (Monday, January, season, Antiquity). For these items, it is reasonable to assume that a spatial or temporal representation is the most salient aspect of the word's meaning. This was the case for only two of the object words (22\% of targets) but for four of the event words (44\% of targets). Therefore, overall, it seems likely that any asymmetry in conceptual salience favored the temporal meanings of the event words, thus working against the hypothesized space-time asymmetry.

These results suggest that the asymmetric dependence of time on space in psychophysical judgments is not an artifact of perceptual or conceptual asymmetries built into the stimuli. Rather, this performance asymmetry reflects a fundamental difference in the way people mentally represent space and time. Yet, this asymmetric relationship between space and time in the mind may, indeed, result from an asymmetry in how perceptible space and time are more broadly -- not in any particular experimental stimuli, but rather in the observable world, in general. Space and time are correlated in our everyday experiences (e.g., as objects travel farther more time passes), and tracking these correlations may be useful for anticipating changes in the physical environment. Correlation is a symmetrical relationship, but people may rely more heavily on the more perceptually available dimension (space), using it heuristically as an index of changes in the less perceptible dimension (time).

It appears that time and space are, in Garner's [15] terminology, asymmetrically separable dimensions: it is possible to ignore irrelevant variation in time while judging space but not possible (or more difficult) to ignore irrelevant variation in space when judging time. At present, there is nothing in Walsh's [1] ATOM proposal that can predict or explain the asymmetric separability of space and time. Yet, this crossdimensional relationship is readily predicted by metaphor theory.

Importantly, space and time are predicted to be related asymmetrically but not unidirectionally. There is evidence that time can influence space in some paradigms [e.g., 11], just as people can sometimes use temporal words to talk about space (e.g., "I live two minutes from the station" is a temporal metaphor for spatial distance). Simply showing that time can influence spatial judgments in some cases does not challenge the asymmetry we report here: to address the question of asymmetry, the cross-dimensional influences of time and space must be appropriately compared, controlling for salience and discriminability across dimensions, and for withindimension performance. 
We propose that Garner-like tests of dimensional separability will be critical for either modifying ATOM or deciding to abandon it in favor of a metaphorical theory of spatial, temporal, and numerical magnitude representation. In order to understand how space, time, and other prothetic dimensions are represented in the brain and mind, it is necessary to go beyond investigating whether these dimensions interact and determine how they interact.

\section{References}

1. Walsh, V.: A theory of magnitude: common cortical metrics of time, space and quantity. Trends in Cog. Sci. 7(11), 483-488 (2003)

2. Basso, G., Nichelli, P., Frassinetti, F., di Pellegrino, G.: Time perception in a neglected space. Neuroreport 7, 2111-2114 (1996)

3. Church, R.M., Meck, W.H.: The numerical attribute of stimuli. In: Roitblat, H.L., Beaver, T.G., Terrace, H.S. (eds.) Animal Cognition, pp. 445-464. Erlbaum, Mahwah (1984)

4. Fischer, M.: Cognitive representation of negative numbers. Psych. Sci. 14(3), 278-282 (2003)

5. Gallistell, R.C., Gellman, R.: Non-verbal numerical cognition: from reals to integers. Trends in Cog. Sci. 4, 59-65 (2000)

6. Cappelletti, M., Freeman, E.D., Cipolotti, L.: Dissociations and interactions between time, numerosity and space processing. Neuropsychologia 47(13), 2732-2748 (2009)

7. Lakoff, G., Johnson, M.: Philosophy in the flesh: The embodied mind and its challenge to Western thought. University of Chicago Press, Chicago (1999)

8. Clark, H.H.: Space, time, semantics and the child. In: Moore, T.E. (ed.) Cognitive Development and the Acquisition of Language, pp. 27-63. Academic Press, New York (1973)

9. Boroditsky, L.: Metaphoric structuring: understanding time through spatial metaphors. Cognition 75(1), 1-28 (2000)

10. Casasanto, D., Fotakopoulou, O., Boroditsky, L.: Space and Time in the Child's Mind: Evidence for a Cross-Dimensional Asymmetry. Cog. Sci. 34, 387-405 (2010)

11. Casasanto, D., Boroditsky, L.: Time in the Mind: Using space to think about time. Cognition 106, 579-593 (2008)

12. Santiago, J., Román, A., Ouellet, M.: Flexible foundations of abstract thought: A review and a theory (submitted)

13. Pansky, A., Algom, D.: Stroop and Garner effects in comparative judgement of numerals: The role of attention. JEP:HPP 25, 39-58 (1999)

14. Garner, W.R.: Interaction of Stimulus Dimensions in Concept and Choice Processes. Cog. Psych. 8, 98-123 (1976)

15. Ornstein, R.: On the experience of time. Penguin, Hammondsworth (1969)

16. Miles, L.K., Nind, L.K., Macrae, C.N.: Moving Through Time. Psych. Sci. (2010), doi: $10.1177 / 0956797609359333$ 